\title{
Tratamento cirúrgico de epilepsia refratária da área da língua
}

\author{
Arthur Cukiert*, Elcio Machado**, Alcione Sousa*, José A. Buratini**, Joaquim Vieira**, \\ Casio Forster ${ }^{* * *}$, Viviane B. Ferreira**, Meire Argentoni**, Leila Frayman ${ }^{* * *}$, Guilherme \\ Malacarne ${ }^{* * *}$
}

Serviço de Cirurgia de Epilepsia do Hospital Brigadeiro e Clínica de Epilepsia de São Paulo, SP

\section{RESUMO}

O presente estudo relata um paciente com foco epiléptico localizado nas áreas motoras e sensitivas da língua, investigado de maneira invasiva com eletrodos subdurais e, subseqüentemente, submetido à ressecção cortical. O paciente de 22 anos, sexo masculino, possuía crises com versão consciente da cabeça para a esquerda, evoluindo, eventualmente, para crises tônico-clônicas, diárias, ocorrendo em vigília e no sono, desde os 5 anos de idade, refratárias ao tratamento clínico. Monitorização videoeletroencefalográfica revelou surtos interictais de ondas lentas com projeção na região frontocentral (C4-T4). O registro ictal sugeria início na mesma região. A ressonância magnética não revelou alterações. Foi submetido ao implante de grades de eletrodos. O registro eletrocorticográfico crônico com eletrodos subdurais mostrou início das descargas nas regiões motora e sensitiva da língua com espraiamento para a região da face. As descargas intercríticas estendiam-se anterior e posteriormente aos giros rolândicos, bem como ao giro temporal superior. A estimulação cortical da área sensitiva da língua reproduziu as crises clínicas. Foi realizada ressecção subpial do córtex pré-motor, motor e sensitivo da língua e face e póssensitiva da língua e face e porções do giro temporal superior, com especial atenção para a preservação do arcabouço vascular da região. Mantém-se sem crises a partir daí. O estudo anatomopatológico demonstrou apenas gliose intensa.

O tratamento cirúrgico de pacientes com focos epilépticos em região da língua pode ser realizado com segurança desde que as relações entre as áreas eloqüentes e epileptogênicas sejam bem conhecidas, em casos selecionados.

\section{PALAVRAS-CHAVE}

Epilepsia. Áreas motora e sensitiva de língua. Cirurgia de epilepsia.

\section{ABSTRACT}

Epileptogenic area in the sensorimotor cortical representation of tongue. Case report treated by surgery

We report the case of a 22 year-old male patient with an epileptic focus located at the sensorimotor area of the tongue who was evaluated with subdural electrodes and submitted to a subpial cortical resection. He had seizures characterized by head rotation to the left without impairment of consciousness. They occurred in a daily basis, either during sleep or awake, since the age of 5 years. Video-EEG monitoring showed interictal slow waves over the right fronto-central region. Ictal recording suggested the onset over the same area. MRI was normal.

He was submitted to subdural electrodes implantation over the right frontoparietal region. ECoG obtained through the implanted electrodes showed ictal onset over the motor and sensitive areas of the tongue with early spreading to the face areas. Interictal spiking was more widespread including the premotor gyrus, the postcentral gyrus and the superior temporal gyrus. Cortical stimulation of the tongue areas reproduced the patient's habitual seizures. A subpial resection of the premotor, motor, sensory and postsensory cortex related to the tongue and an additional superior temporal gyrus resection was performed. Special caution was dedicated to the preservation of the surrounding vascular structures. He has been seizure-free since surgery. Pathological examination showed severe gliosis.

The surgical treatment of patients with foci at the tongue area can be performed safely as far as the relationship between the focus and the surrounding eloquent areas is well delimited.

\section{KEYWORDS}

Epilepsy. Sensorimotor areas of tongue. Epilepsy surgery.

\footnotetext{
* Chefe do Serviço.

** Neurocirurgiões.

*** Neurofisiologistas.

**** Estagiário.
} 


\section{Introdução}

A neurofisiologia do córtex cerebral relacionada à representação motora e sensitiva da língua tem sido extensamente estudada nos últimos 50 anos. Há uma clara organização somatotópica do córtex motor e sensitivo primários, uma vez que a região da língua e da face estende-se por cerca de $3 \mathrm{~cm}$ acima da fissura silviana nos giros pré e pós-central. Há maior representação da língua no homúnculo motor do que no sensitivo. Áreas de grande sensibilidade tátil e maior densidade de inervação periférica possuem representações corticais relativamente maiores. O mapeamento do córtex lingual é útil na determinação do sulco central e de seu ponto de aproximação com a fissura silviana?

Diversos métodos de mapeamento cortical intra e pré-operatórios, como eletrocorticografia e eletroestimulação cortical ${ }^{5,8}$, ultra-sonografia ${ }^{5}$ e registro de potenciais evocados ${ }^{1,4,10}$, já foram usados com o objetivo de melhor precisar as regiões-alvo. A história e o exame clínico do paciente, investigações eletroencefalográficas, avaliação das características da crise, estudos neuropsicológicos e de neuroimagem ${ }^{9}$ também oferecem subsídios para o diagnóstico e o tratamento.

Movimentos involuntários da língua podem ser vistos em diferentes enfermidades. Síndrome mastigatória bucolingual por uso de drogas neurolépticas, doença de Huntington e distonias oromandibulares são alguns exemplos. Esses movimentos anormais são, de maneira geral, contínuos ${ }^{6}$. Descargas epilépticas no córtex da parte inferior das áreas somatossensitiva e motora produzem manifestações motoras e sensitivas envolvendo a língua. O primeiro relato de movimentos linguais como manifestação de descargas epilépticas focais foi descrito por Holtzman, em 1984, causados por um meningeoma de convexidade ${ }^{2}$.

O presente estudo relata um paciente com foco epiléptico localizado nas áreas motoras e sensitivas da língua, investigado de maneira invasiva com eletrodos subdurais e, subseqüentemente, submetido à ressecção cortical.

\section{Relato do caso}

ROA, 22 anos, sexo masculino, possuía crises com versão consciente da cabeça para a esquerda, evoluindo eventualmente para crises tônico-clônicas, diárias, ocorrendo em vigília e no sono, desde os 5 anos de idade, refratárias ao tratamento clínico. Monitorização videoeletroencefalográfica revelou surtos interictais de ondas lentas com projeção na região frontocentral (C4T4). O registro ictal sugeria início na mesma região. As imagens obtidas no SPECT ictal demonstraram hiperfluxo temporal direito. A ressonância magnética não revelou alterações.

Foi submetido ao implante de grades de eletrodos na convexidade e na região mesial frontoparietal por meio de uma extensa craniotomia frontoparietal direita (Figura 1). O registro eletrocorticográfico crônico mostrou início das descargas nas regiões motora e sensitiva da língua com espraiamento para a região da face (Figura 2). As descargas intercríticas estendiam-se anterior e posteriormente aos giros rolândicos, bem como ao giro temporal superior. A estimulação cortical da área sensitiva da língua reproduziu as crises clínicas (Figura 3). A seguir, foram retirados os eletrodos e realizada ressecção subpial do córtex pré-motor, motor e sensitivo da língua e da face e pós-sensitiva da língua e da face e porções do giro temporal superior, com especial atenção para a preservação do arcabouço vascular da região (Figura 4). Apresentou discreta paralisia facial à esquerda, que regrediu em 1 mês. Mantém-se sem crises a partir daí. $\mathrm{O}$ estudo anatomopatológico demonstrou apenas gliose severa.

\section{Discussão}

A estimulação cortical direta é um bom método para analisar a localização de áreas corticais específicas. Problemas técnicos relacionados ao procedimento incluem a presença de áreas corticais silenciosas e não responsivas e a influência não-seletiva da atividade neuronal de áreas subjacentes ${ }^{7}$. Além disso, parte do córtex central está escondido nas paredes dos sulcos, e a superfície explorada representa pouco mais de um terço da área funcional. Desenvolvimentos técnicos recentes têm contribuído para a superação de alguns desses problemas.

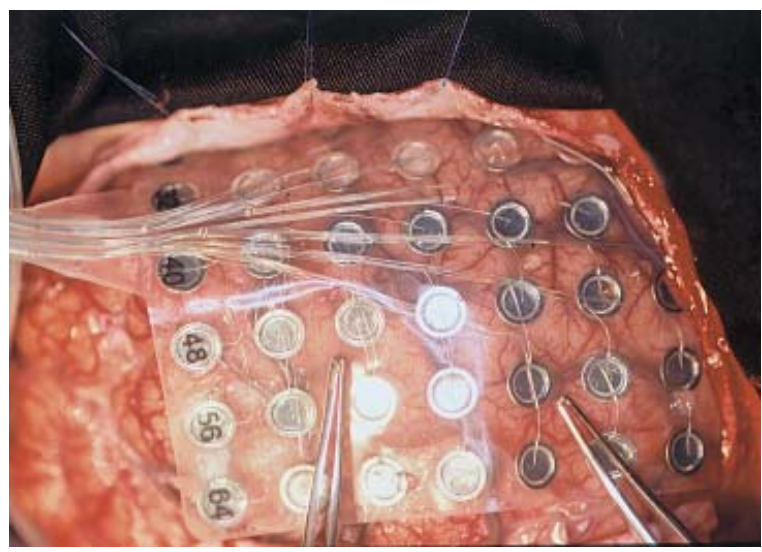

Figura 1 - Visão intra-operatória mostrando eletrodos em posição, centrados nas áreas rolândicas. 


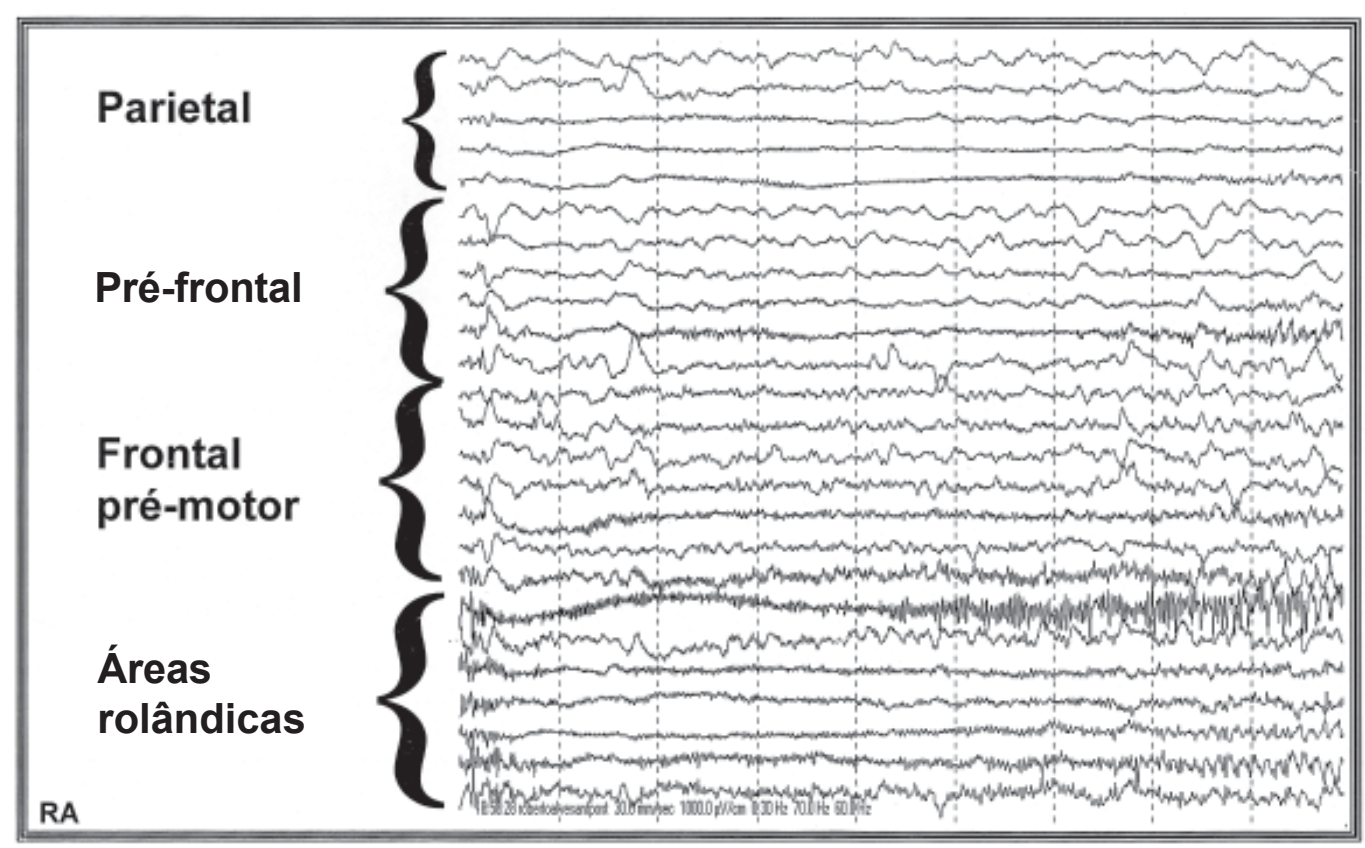

Figura 2 - Eletrocorticografia crônica mostrando início crítico nas regiões rolândicas correspondentes à língua.

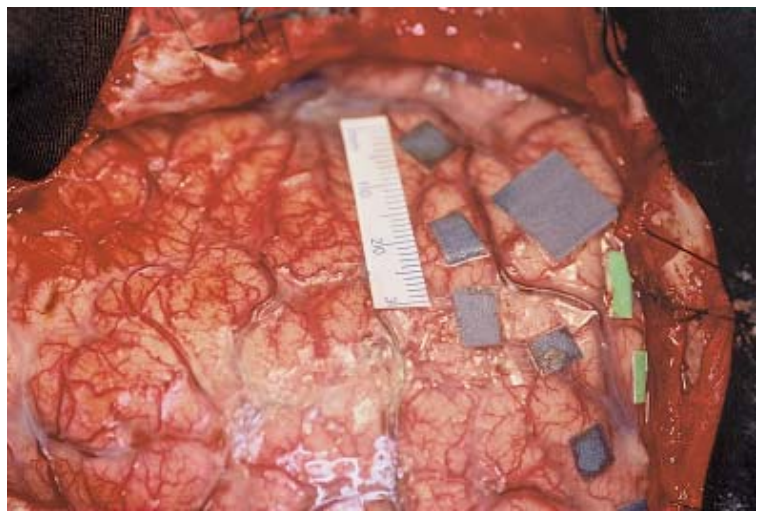

Figura 3 - Visão intra-operatória mostrando o resumo dos achados neurofisiológicos. Etiquetas azuis pequenas: giro motor. Etiqueta azul grande: área sensitiva da língua, próxima à fissura silviana, a partir da qual obteve-se a crise típica do paciente por meio de estimulação cortical.

Etiquetas verdes pequenas: giro sensitivo. A régua demarca a distância de $3 \mathrm{~cm}$ ao longo do giro motor, a partir da fissura silviana.

Há uma clara organização somatotópica nos córtices rolândicos. A extremidade da língua está representada superiormente e, a sua parte posterior, mais caudalmente nos giros pré e pós-central. A extremidade, ou porção anterior da língua, é mais amplamente representada que as partes média e posterior da mesma. Isso se deve, possivelmente, à relação da parte anterior da língua com a fala, seu papel funcional mais relevante. $\mathrm{O}$ hemisfério dominante para a fala contém maior representação cortical para a língua ${ }^{7}$. Algum grau de assimetria pode ocorrer na contração da musculatura craniofacial de acordo com a dominância cerebral, a despeito da bilateralidade da inervação corticobulbar.

A grande concentração das respostas linguais à estimulação elétrica imediatamente acima da fissura silviana torna mais fácil a exata determinação do ponto de aproximação do sulco central com a mesma. Esse marco é de grande importância para a prevenção de disfasia pós-operatória quando se planeja a ressecção frontal ou temporal no hemisfério dominante para a fala.

Movimentos involuntários da língua têm sido relatados durante o tratamento com drogas antiepilépticas ou como parte de complexas discinesias orofaciais ocorrendo durante intoxicação medicamentosa ${ }^{3}$. Da mesma forma, movimentos linguais por desordens de gânglios basais são complexos e ocorrem em conjunto com movimentos de estruturas adjacentes. Tremores da língua são observados no alcoolismo crônico e na doença de Parkinson. Ocasionalmente, tiques e mioclonias da língua são vistos como parte de uma desordem mais ampla.

Descargas epilépticas caracterizadas por movimentos linguais têm sido raramente documentadas na literatura. Holtzman (1984) ${ }^{2}$ descreveu um caso de epilepsia lingual em uma paciente de 63 anos. As crises iniciavam-se como uma sensação de inchaço e alargamento da hemilíngua esquerda, seguida por percepções disestésicas na mucosa oral. Subseqüentemente, ocorriam movimentos clônicos da língua que eram sucedidos por sensações de disfagia e impossibilidade para falar ou emitir sons. As crises evoluíam 


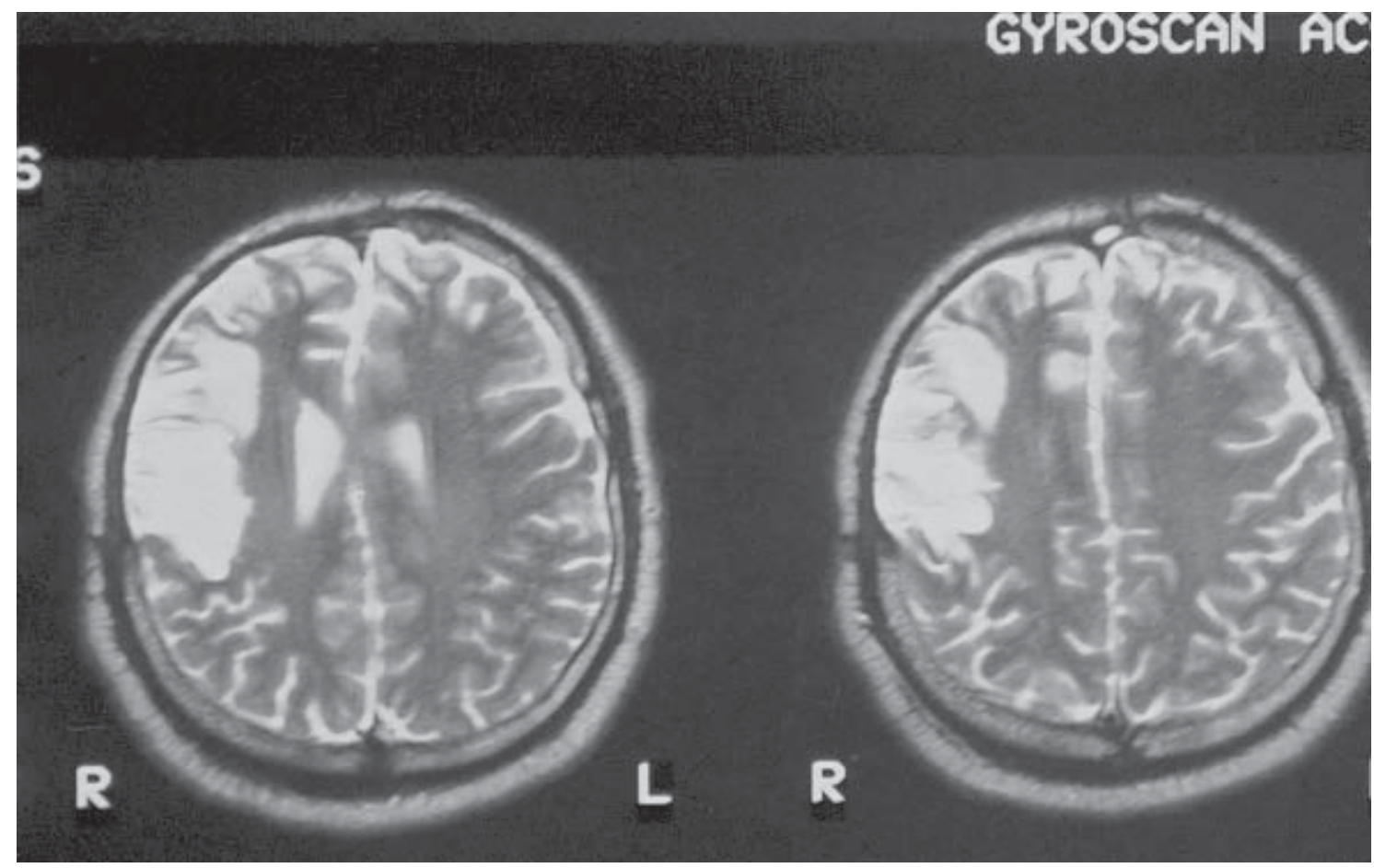

Figura 4 - RM pós-operatória em T2 mostrando a ressecção subpial central realizada.

posteriormente com movimentos versivos da cabeça para a esquerda e clônicos da musculatura facial à esquerda. Tomografia de crânio diagnosticou um meningeoma sobre o giro sensitivo à direita. As crises cessaram após a ressecção do tumor.

Neufeld $(1988)^{6}$ relatou um paciente de 73 anos com movimentos clônicos involuntários da língua, precedidos por salivação e associados com dor lancinante na base da língua. Em algumas ocasiões, ocorria versão ocular e cefálica para a esquerda e contrações clônicas do canto esquerdo da boca. Traçados eletroencefalográficos ictais demonstraram atividade semi-rítmica de 3 a $5 \mathrm{~Hz}$ intercalada com ondas agudas nas regiões frontocentrais direitas. As crises responderam satisfatoriamente ao tratamento clínico.

O presente paciente apresentava crises refratárias com versão cefálica para a esquerda e contrações segmentares tônico-clônicas do mesmo lado. Tais crises deviam-se ao espraiamento das descargas provindas das regiões corticais sensitivas e motoras relativas à língua para as regiões da face. A ressecção cirúrgica subpial das áreas epileptogênicas, com especial atenção para a preservação do suprimento vascular proveniente da fissura silviana, culminou com o desaparecimento das crises e ausência de morbidade neurológica. A lesão do arcabouço vascular arterial e venoso conduz a um aumento inaceitável da morbidade cirúrgica.

O tratamento cirúrgico de pacientes com focos epilépticos na região correspondente à da língua pode ser realizado com segurança desde que as relações entre as áreas eloqüentes e epileptogênicas sejam bem conhecidas. Quando realizado no hemisfério dominante, cuidado ainda maior deve ser exercido com o arcabouço vascular para evitar-se lesão ou disfunção das áreas da fala adjacentes.

\section{Referências}

1. GREGOIRE EM, GOLDRING MD: Localization of function in the excision of lesions from the sensorimotor region. J Neurosurg 61:1047-54, 1984.

2. HOLTZMAN RNN, MARK MH, WIENER LM, MINZER L: Lingual epilepsy: a case report of an unusual expression of focal cerebral discharge. J Neurol Neurosurg Psychiatry 47:317-8, 1984.

3. JABBARI B, COKER SB: Paroxymal, rhytmic lingual movements and chronic epilepsy. Neurology 31:13647, 1981.

4. KING RB, SCHELL MD: Cortical localization and monitoring during cerebral operations. J Neurosurg 67:210-9, 1987

5. Le ROUX PD, BERGER MS, HAGLUND MM, PILCHER WH, OJEMANN GA: Resection of intrinsic tumors from nondominant face motor cortex using stimulation mapping: report of two cases. Surg Neurol 36:44-8, 1991.

6. NEUFELD MY, BLUMEN SC, NISIPEANU P, KORCZYN AD: Lingual Seizures. Epilepsia 29:30-3, 1988.

7. PICARD C, OLIVIER A: Sensory cortical tongue representation in man. J Neurosurg 59:781-9, 1983. 
8. UEMATSU S, LESSER R, FISHER R, KRAUSS G, HART J, VINNING EP, FREEMAN J, GORDON B: Resection of the epileptogenic area in critical cortex with the aid of a subdural electrode grid. Stereotact Funct Neurosurg 55:34-45, 1990.

9. WILLIAMSON PD, THADANI VM, DARCEY TM, SPENCER DD, SPENCER SS, MATTSON RH: Occipital lobe epilepsy: clinical characteristics, seizure spread patterns and results of surgery. Ann Neurol 31:3-13, 1992.

10. WOOD CC, SPENCER DD, ALLISON T, McCERTHY G. WILLIAMSIN PD, GOFF WR: Localization of human sensorimotor cortex during surgery by cortical surface recording of somatosensory evoked potentials. J Neurosurg 68:99-111, 1988.

Original recebido em agosto de 2000

Aceito para publicação em outubro de 2000

\section{Endereço para correspondência:}

Arthur Cukiert

Rua Dr. Alceu Campos Rodrigues, 247, 12adar, cj. 121 CEP 04544-000 - São Paulo, SP

E-mail:acukiert@uol.com.br 